\title{
SOME PROPERTIES OF QUADRATIC WORDS IN FREE GROUPS
}

\section{CHARLES C. EDMUNDS}

ABSTRACT. In a free group, two quadratic words have the same set of endomorphic images if and only if they have the same set of automorphic images. This is not the case for arbitrary words.

1. Introduction. Let $g$ be an element of a group $G$ and let $E_{g}$ (resp. $A_{g}$ ) denote the set of endomorphic (resp. automorphic) images of $g$ in $G$. Let $F$ be the countably generated free group $\left\langle x_{1}, x_{2}, \cdots ; \varnothing\right\rangle$ and call $w \in F$ a quadratic word if each $x_{i}$ occurring in $w$ appears, with exponent +1 or -1 , exactly twice.

If $g, b \in G$ and $A_{g}=A_{b}$, it is clear that $E_{g}=E_{b}$; however the converse is false. With $G=F_{2}\left(=\left\langle x_{1}, x_{2} ; \varnothing\right\rangle\right), g=x_{1}$, and $h=x_{1}^{2} x_{2}^{3}$, it is easy to see that $E_{g}=E_{b}=F_{2}$. But $A_{g}=A_{b}$ implies that $b$ is primitive, which is not the case (see $[5, \mathrm{p} .170])$. Thus $x_{1}$ and $x_{1}^{2} x_{2}^{3}$ have the same set of endomorphic images in $F_{2}$, but no automorphism of $F_{2}$ sends $x_{1}$ to $x_{1}^{2} x_{2}^{3}$. It is interesting to note that the situation is different for quadratic words. $A_{h^{*}}$

Theorem. If $g$ and $h$ are quadratic words in $F$ with $E_{g}=E_{h}$, then $A_{g}=$

On the way toward proving this theorem we will derive a new proof of the following result of Roger Lyndon and Morris Newman.

Lemma 1 [4, Theorem 2]. The word $s(n)=x_{1}^{2} x_{2}^{2} \cdots x_{n}^{2}$ cannot be written as the product of fewer than $n$ squares in $F$.

Our other lemma is the analogue for commutators, $[x, y]=x y x^{-1} y^{-1}$.

Lemma 2. The word $c(n)=\left[x_{1}, x_{2}\right]\left[x_{3}, x_{4}\right] \cdots\left[x_{2 n-1}, x_{2 n}\right]$ cannot be written as the product of fewer than $n$ commutators in $F$.

Received by the editors April 29, 1974 . $20 \mathrm{~F} 55$.

AMS (MOS) subject classifications (1970). Primary 20E05; Secondary 20F10, word.

Key words and phrases. Free group, automorphism, endomorphism, quadratic 
We note that Lemma 1 was generalized, in [3], to any product of $n$th powers. This and both lemmas follow from a more general result of A. Rhemtulla [6]; however, we will provide shorter and less complex proofs for Lemmas 1 and 2. All proofs are by word arguments alone in contrast to those given in [4]. The only results used without proof are Theorem $E$ of [2] and an elementary result of M. Dehn [1].

2. Proofs. It was proved in [2] that if $g \in F$ is a quadratic word, there is a finite set of quadratic words $B_{\boldsymbol{g}} \subseteq E_{\boldsymbol{g}}$ which spans $E_{\boldsymbol{g}}$ in the following sense:

For each $h \in E_{g}$, there exists a word $w\left(=x_{i(1)}^{\epsilon(1)} x_{i(2)}^{\epsilon(2)} \cdots x_{i(t)}^{\epsilon(t)}, \epsilon(j)= \pm 1\right)$ in $B_{g}$ and an endomorphism $\phi$ of $F$ such that (i) $w \phi=h$, (ii) $x_{i} \phi \neq 1$ (the empty word) for each $i$, and (iii) no cancellation occurs in forming the product

$$
\left(x_{i(1)}^{\epsilon(1)} \phi\right) \cdot\left(x_{i(2)}^{\epsilon(2)} \phi\right) \cdot \ldots \cdot\left(x_{i(t)}^{\epsilon(t)} \phi\right) .
$$

Proof of Lemma 1. By way of contradiction, suppose that $s(n)$ can be written as the product of $m(<n)$ squares. This implies that $s(n) \in E_{s(m)}$ and that $E_{s(m)}=E_{s(k)}$ for each $k \geq m$. By the result quoted above, $B_{s(m)}$ is a finite set; thus there is an upper bound $L>0$ on the length of the words in $B_{s(m)}$. Since $B_{s(m)}$ spans $E_{s(m)} \supseteq E_{s(L)}$, there is a word $w=x_{i(1)}^{\epsilon(1)} x_{i(2)}^{\epsilon(2)}$ $\cdots x_{i(t)}^{\epsilon(t)} \in B_{s(m)}$ and an endomorphism $\phi$ such that $w \phi=s(L), x_{i} \phi \neq 1$ for each $i$, and no cancellation occurs in forming the product (1).

Since $w \in B_{s(m)}$, the length of $w$ is at most $L$. The length of $s(L)$ is $2 L$; therefore, there is an $r$ such that $x_{i(r)}^{\epsilon(r)} \phi$ has length at least two. Since $w$ is quadratic, $x_{i(r)}=x_{i(s)}$ for some $s \not r ;$ thus the segment $x_{i(r)}^{\epsilon(r)} \phi$, or its inverse, appears twice in $s(L)$. In fact since no negative exponents occur in $s(L)$, it contains two nonoverlapping occurrences of $x_{i(r)}^{\epsilon(r)} \phi$. But no segment of length two occurs more than once in $s(L)$; this is a contradiction.

Proof of Lemma 2. If we suppose that $c(n)$ can be written as the product of $m(<n)$ commutators, reasoning similar to that used in the previous proof shows that $E_{c(k)} \subseteq E_{c(m)}$ for every $k$. Since $c(m)$ is quadratic, $B_{c(m)}$ is finite and there is an upper bound, $L>0$, on the length of the words in $B_{c(m)}$. Since $B_{c(m)}$ spans $E_{c(m)} \supseteq E_{c(L)}$, it follows that there is a $w \in B_{c(m)}$, and an endomorphism $\phi$ such that $w \phi=c(L)$. As in the previous proof, this leads to a contradiction.

Proof of the Theorem. Max Dehn [1] observed that if $g$ is a quadratic word in $F$, there is an automorphism $\alpha$ of $F$ sending $g$ to either $s(n)$ or $c(n)$ for some $n \geq 0$. Since $g$ and $h$ are assumed to be quadratic, there are 
automorphisms $\alpha$ and $\beta$ of $F$ such that $g \alpha=s(m)$ or $c(m)$ and $h \beta=s(n)$ or $c(n)$ for some $m$ and $n$.

By assumption there is an endomorphism $\phi$ such that $g \phi=h$; suppose that $g \alpha=c(m)$ and $h \beta=s(n)$. The endomorphism $\alpha^{-1} \phi \beta$ sends $c(m)$ to $s(n)$ and thus $s(n)$ lies in the commutator subgroup $F^{\prime}$, since $F^{\prime}$ is fully invariant. This is obviously false; therefore, we have either $g \alpha=s(m)$ and $h \beta=s(n)$ or $g \alpha=c(m)$ and $h \beta=c(n)$.

By Lemma $1, s(m)$ and $s(n)$ are endomorphic images of each other if and only if $m=n$. By Lemma 2, the same holds for $c(m)$ and $c(n)$. Thus $g$ and $h$ are endomorphic images of each other if and only if $g \alpha=h \beta$. It follows that $A_{8}=A_{h}$.

\section{REFERENCES}

1. M. Dehn, Über unendliche diskontinuierliche Gruppen, Math. Ann. 71 (1912), $116-144$.

2. C. Edmunds, On the endomorphism problem for free groups, Comm. Algebra 3 (1975), 1-20.

3. R. Lyndon, T. McDonough and M. Newman, On products of powers in groups, Proc. Amer. Math. Soc. 40 (1973), 419-420. MR 47 \#8693.

4. R. Lyndon and M. Newman, Commutators as products of squares, Proc. Amer. Math. Soc. 39 (1973), 267-272. MR 47 \#3546.

5. W. Magnus, A. Karrass and D. Solitar, Combinatorial group theory: Presentations of groups in terms of generators and relations, Pure and Appl. Math., vol. 13, Interscience, New York, 1966. MR 34 \#7617.

6. A. Rhemtulla, A problem of bounded expressibility in free products, Proc. Cambridge Philos. Soc. 64 (1968), 573-584. MR 37 \#1480.

DEPARTMENT OF MATHEMATICS AND ASTRONOMY, UNIVERSITY OF MANITOBA, WINNIPEG, MANITOBA, CANADA R3T 2N2 